\title{
Morphologische und ökologische Grundlagen der Vorlandsicherung durch Puccinellia maritima (Gramineae)
}

\author{
K. VON WEIHE \\ Institut für Angewandte Botanik der Universität Hamburg, \\ Marseiller Str. 7, D-2000 Hamburg, Bundesrepublik Deutschland
}

\begin{abstract}
Morphological and ecological principles of the coastal land-preservation by Puccinellia maritima (Gramineae). Investigations on the influence of daylength, light intensity, inundation and accretion tolerance on the morphological and ecological characteristics of Puccinellia maritima in connection with the role of increasing salinity and temperature (results of former investigations) lead to a deeper understanding of the role this species plays in land reclamation and preservation of coastal areas. The results obtained indicate that the species exhibits a temperature related seasonal dimorphism with short-stem growth at daylengths up to about $12 \mathrm{~h}$ light/day (vernal and autumnal types, caespitose growth) and a long-stem growth at daylength of about $16 \mathrm{~h}$ and more light/day (aestival type, stoloniferous growth). Increasing salinity (above $10 \% \mathrm{~S}$ ) and inundation with artificial sea water $(25 \% \mathrm{~S})$ reduce stem and leave development. Accretion of long-stems with sand $+3 \%$ peat-mixture up to $30 \mathrm{~cm}$ yields in $5 \% \mathrm{~S}$ further development of runners under long-day conditions and longation of short-stems (up to $10 \mathrm{~cm}$ ) up to the new soil-surface level. Three shortstem types and two long-stem types can be distinguished. These results explain the natural responses of the species, the forming of the Puccinellietum maritimae within the allogene succession and its role in land preservation. The species is a facultative halophyte which occupies, due to competition from other species, the lower part of its inundation amplitude, utilising its high-salt tolerance. Its aestival long-stem and its autumnal/vernal short-stem production increases the sedimentation rate of soil particles during inundation; the following accretion leads to an elongation of short-stems up to the new soil level, and to an increased production of adventitious roots; the latter results in soil fixation.
\end{abstract}

\section{EINLEITUNG}

Der Andel, Puccinellia maritima (Huds.) Parl. (= P. maritima), spielt für die biogene Landgewinnung, die Vorlandsicherung und den Küstenschutz u. a. im Bereich der deut-

NT-See-Küsten eine hervorragende Rolle (Wohlenberg, 1969), da durch diese ausdauernde Art die Ausbildung der bodendeckenden und bodensichernden unteren Salzwiese im Meerwassertidebereich mit der Ausbildung des Puccinellietum maritimae einsetzt.

Die Besiedlung dieser Extremstandorte und die Rolle bei der ablaufenden allogenen Sukzession stehen in engem Zusammenhang mit den morphologischen und ökologischen Merkmalen der Art. Dieses Zusammenwirken morphologisch-ökologischer Merkmale wurde zuerst von Wohlenberg $(1933,1931)$ bei der Beobachtung der Ausbildung der 
"Andelpolster" im Bereich der Grünen Insel der Eidermündung erkannt, die die Grundlage für die Bodenfestlegung und z. T. für den Schlickfang darstellen (vgl. auch Ranwell, 1975).

Voraussetzung hierfür ist einerseits die Ausbildung von oberirdischen Ausläufern, ein differentialdiagnostisches Merkmal der Art (Hudson, 1762; Dreyling, 1973), die an brackischen natürlichen Standorten beträchtliche Längen (z. B. Schratz \& Beiler, 1937) und in Vegetationsversuchen bis zu $160 \mathrm{~cm}$ (von Weihe, 1978), bei Einzelisolierungen bis über $200 \mathrm{~cm}$ (Petermann, 1970) erreichen können; andererseits ist eine entsprechend breite Amplitude der Meersalzverträglichkeit und eine entsprechende Ưberflutungsverträglichkeit Voraussetzung (von Weihe \& Dreyling, 1970).

$P$. maritima weist einen ausgeprägten Saisondimorphismus mit einer Ausläuferbildung in den Sommermonaten und horstförmigem Wachstum in den Herbst- und Frühjahrsmonaten auf, der bei der Bodenaussüßung die Ursache für eine Konkurrenzschwäche im Wettbewerb mit Festuca rubra, Lolium perenne und Poa pratensis darstellt (von Weihe, 1969).

Das Zusammenwirken dieser Merkmale mit den ökologischen Faktoren am natürlichen Standort ist vermutlich die Grundlage der Ausbildung eines Puccinellietum maritimae mit dem Verbreitungsschwerpunkt von $P$. maritima in Höhe und wenige Dezimeter oberhalb der Mittleren Tidehochwasserlinie (= MThw) und Gegenstand der hier vorgelegten experimentellen Untersuchung. Ein Teil dieser Endergebnisse ist bei der Ausarbeitung der Methodik zur Ermittlung der Salz- und Uberflutungsverträglichkeit (von Weihe \& Dreyling, 1970) verwendet worden.

\section{MATERIAL UND METHODIK}

\section{Material und morphologische Grundlagen}

Für alle nachfolgenden Untersuchungen wurden Isolierungen von Puccinellia maritima Sortiment-Nr. 57 aus der Eidermündung (nö. vom Schülper Siel) benutzt, die im Jahre 1964 aus der Optimalphase des Puccinellietum maritimae in Sodenform entnommen und auf dem Versuchsfeld des Institutes unter glykophytischen Bedingungen weiterkultiviert und $z$. T. in Frühbeetkästen bzw. im Gewächshaus vegetativ vermehrt wurden.

Alle hier verwendeten Isolierungen sind der f. vulgaris Dreyling (1973) zuzuordnen, die nach von Weihe \& Dreyling (1970) und von Weihe (1978) mehrere Salinitätstypen umfaßt. Isolierungen aus dieser Provenienz Sort.-Nr. 57 sind für alle grundlegenden Untersuchungen an P. maritima des Hamburger Institutes seit dem Jahre 1964 verwendet worden.

P. maritima (Huds.) Parl. f. vulgaris Dreyling ist mit ihrer eigenen morphologischen und ökologischen Mannigfaltigkeit u. a. durch die Ausprägung langer oberirdischer Ausläufer gegen die f. arenaria (Fries) Holmb. und die f. americana (Sør.) Dreyling nach Dreyling (1973) abgegrenzt. Die f. vulgaris bildet saisonabhängig orthotrope und plagiotrope Langtriebe aus, die topographisch bedingt zu Kriechtrieben bzw. zu oberirdischen Ausläufern werden (Abb. 1). Achselknospen der Ausläuferblätter können z. T. saisonabhängig wiederum Langtriebe oder auch Kurztriebe mit reicher Bestockung ausbilden 

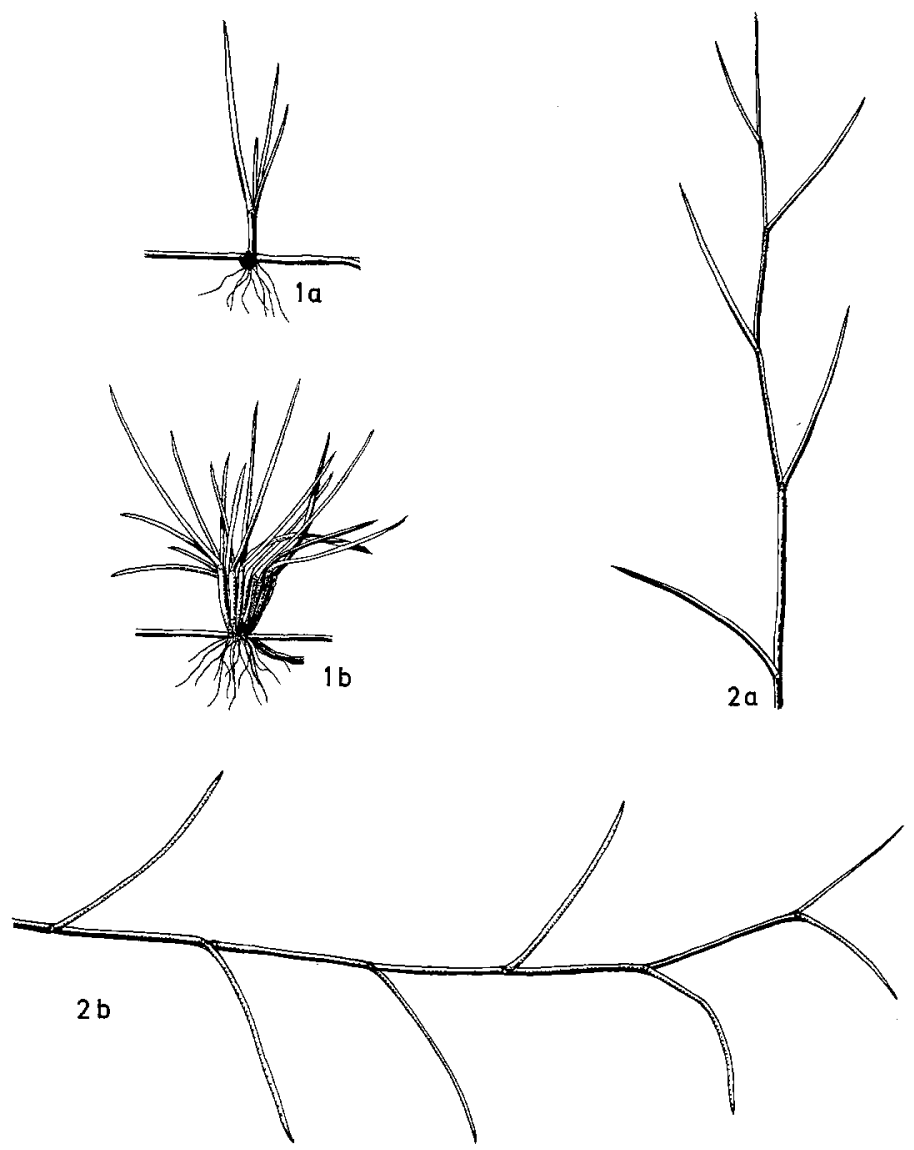

Abb. 1: Sproß-Typen von Puccinellia maritima. 1a: Kurztrieb ohne Bestockung, 1b: Kurztrieb mit Bestockung, 2a: orthotroper Langtrieb, 2b: plagiotroper Langtrieb

(Petermann, 1970). Die entwickelten Langtriebe umfassen zahlreiche Knoten und \pm lange Internodien; die proximalen Blätter sterben früher oder später $a b$, so daß im distalen Bereich z. B. ca. 5-7 grüne Blätter verbleiben; an den Knoten entwickeln sich meistens in Zusammenhang mit der Bestockung bei Bodenauflage oder Übersandung bzw. Uberschlickung Adventivwurzeln. Proximale Abschnitte oder bisweilen auch sämtliche Internodien der plagiotropen Langtriebe sterben ebenso wie der größte Teil orthotroper Langtriebe in den Wintermonaten ab.

Hieraus resultiert im Prinzip folgender vegetativer Vermehrungsrhythmus: Langtriebe (Sommermonate) - Kurztriebe (Herbstmonate ) - Absterben der Internodien der Langtriebe (Wintermonate) - Kurztriebe (Frühjahrsmonate) - Langtriebe (Sommermonate) etc. (s. auch von Weihe, 1969) und folgende prinzipielle Sproßtypisierung bzw. Wuchsformen der Pflanze:

(1a) Kurztrieb ohne Bestockung: horstförmig

(1b) Kurztrieb mit Bestockung: horstförmig 

(2a) orthotroper Langtrieb:
ausläuferförmig
(2b) plagiotroper Langtrieb:
ausläuferförmig

Zwischen diesen Typen treten Ubergänge auf, ihre Ausprägung sowie die SproßTypen selbst sind z. T. Grundlage für die infraspezifische Mannigfaltigkeit, u. a. bei der Abgrenzung der f. americana gegen $f$. vulgaris und f. arenaria (Dreyling, 1973) und für die Rassen- bzw. Okotypen-Charakterisierung (Gray \& Scott, 1975).

\section{Versuchs- und Analysenmethoden}

Im Prinzip wurden alle Versuche nach der Methodik früherer Untersuchungen (von Weihe, 1963) bzw. entsprechend der grundlegenden methodischen Bearbeitung von v. Weihe \& Dreyling (1970) durchgeführt. Hiernach wurde $P$. maritima nach entsprechender 8wöchiger Vorkultur im Gieß- oder Flutverfahren in künstlichem Meerwasser ( $=\mathrm{KM}$ ) nach Levring (1946) mit Zusatz einer Grundnährlösung (= GN) nach Henze, einer Eisenkomplexlösung (Jacobson, 1951) und einer A-Z-Lösung Typ "a" nach Hoagland kultiviert. Die speziellen Versuchsanstellungen waren wie folgt:

Die Versuche zur Überprüfung der Wirkung der $\mathrm{T}$ a g e s li cht $\mathrm{d}$ a u e $\mathrm{r}$ und der $\mathrm{L}$ i chtintensität wurden in Klimakammern nach dem Verfahren von v. Weihe (1963) unter Leuchtstofflampen bei $20^{\circ} \mathrm{C}$ Lufttemperatur und $70 \%$ relativer Luftfeuchte mit einer Versuchsdauer von $56 \mathrm{~T}$ agen im Gießverfahren mit den Meersalzkonzentrationen $0,10,20,30 \% \mathrm{KM}$ und jeweils bei einer mittleren Lichtintensität in Höhe des Topfrandes von 5600 Lux und 15500 Lux durchgeführt.

Die Kultur erfolgte in paraffinierten Ton- oder in Plastiktöpfen $(13 \mathrm{~cm} \varnothing)$ mit Hirschauer Quarzkies 1-2 mm + 3\% Flora-Torf (Anordnung vgl. von Weihe, 1963). Ausgewertet wurden Frisch- und Trockengewichtszuwachs der oberirdischen Pflanzenteile sowie Länge und Morphologie der Triebe.

Die Versuche zur Bestimmung der Wirkung unterschiedlicher Uberflu$\mathrm{t} u \mathrm{ng} \mathrm{s}$ d a u e $\mathrm{r}$ wurden entsprechend dem Verfahren von v. Weihe \& Dreyling (1970) in Flutbecken angesetzt. Außer dem dort beschriebenen Quarzkies-Torf-Substrat wurde in diesen Versuchen Seeschlick (entnommen vor Süderhafen der Insel Nordstrand) verwendet. Als Flutwasser wurde $25 \% \mathrm{KM}+\mathrm{GN}$ benutzt. Die Kultur erfolgte im Gewächshaus über einen Zeitraum von 96 Tagen (= 192 Überflutungen). Ausgewertet wurde Frisch- und Trockengewichtszuwachs der oberirdischen Pflanzenteile sowie Anzahl, Länge und Morphologie der Triebe.

Die Übers andungsvers u che wurden im Gewächshaus vorerst im Gießverfahren, bei den Hauptversuchen im Flutverfahren in Anlehnung an von Weihe $\&$ Reese (1968) und von Weihe \& Dreyling (1970) in Mitscherlich-Gefäßen bei einer Konzentration von $5 \% \mathrm{KM}$ des Flutwassers (Versuchsgesamtdauer: 192 Tage) durchgeführt. Die Hauptversuche liefen zum größten Teil im Langtag ( 18-14 Std. Licht/d). Die Kultur und Ubersandung erfolgte mit dem Quarzkies-Torf-Gemisch der obigen Versuche in der Form, daß das Pflanzsubstrat mit einem Kunststoffgewebe (Maschenweite ca. 0,8 mm) vor dem Bepflanzen derart unterfangen wurde, daß nach beliebiger Kulturdauer ein Teil des Substrates höher oder tiefer in den Mitscherlich-Gefäßen neu eingesetzt werden konnte. Den Mitscherlich-Gefäßen wurden entsprechend abgedichtete Kunststoff-Manschetten 
aufgesetzt, um Ubersandungshöhen bis zu $30 \mathrm{~cm}$ zu ermöglichen. Die Fluthöhe war in jedem Falle die eingestellte Substratoberfläche; die erforderlichen Höhen wurden durch entsprechende Verstärkung der Pumpenleistungen und Flutwasservorratsbehälter im Vergleich zu dem zur Bestimmung der Salzverträglichkeit gebrauchten Verfahren erreicht. Ausgewertet wurden Frisch- und Trockengewicht der oberirdischen Pflanzenteile sowie Länge und Morphologie der übersandeten Triebe.

Das für die Übersandung benutzte Quarzkies-Torf-Gemisch wies ein Porenvolumen (= max. Wasserkapazität) von 41,3 Vol.- $\%$ und eine Feldkapazität (Wasserkapazität) von 29,2 Vol.- $\%$ auf.

\section{EXPERIMENTELLER TEIL}

\section{Tageslichtdauer und Lichtintensität}

Die Versuche zur Ermittlung der Wirkung unterschiedlicher Tageslichtdauer und Lichtintensität auf den Zuwachs der oberirdischen Pflanzenteile von Puccinellia maritima ergaben (Abb. 2) - wie bekannt - bei allen Kulturen eine Abnahme der Zuwachsleisturig mit steigender KM-Konzentration > $10 \%$. Hohe Lichtintensitäten führten zu einer glauken Färbung der Pflanzen und zu größerer Festigkeit aller Triebe, die damit der Morphologie der Pflanzen des natürlichen Standortes ähnlicher als die der bei niedriger Lichtintensität kultivierten Pflanzen waren.

Tabelle 1

Mittlere Frischgewichte (g/Versuchsgefäß) von Puccinellia maritima (Sort.-Nr. 57) in Abhängigkeit von der Tageslänge, der Lichtintensität und der Meersalzkonzentration der Gießlösung nach 56tägiger Kultur bei $20^{\circ} \mathrm{C}$ Luftemperatur und $70 \%$ rel. Luftfeuchte in einer Klimakammer

\begin{tabular}{|c|c|c|c|c|c|c|c|c|}
\hline \multirow{2}{*}{$\begin{array}{l}\text { Belichtung } \\
\text { Std/Tag } \\
\text { Lux } \\
\end{array}$} & \multicolumn{2}{|c|}{10} & \multicolumn{2}{|c|}{16} & \multicolumn{2}{|c|}{19} & \multicolumn{2}{|c|}{24} \\
\hline & 5600 & 15500 & 5600 & 15500 & 5600 & 15500 & 5600 & 15500 \\
\hline \multicolumn{9}{|l|}{$\%$ KM } \\
\hline 0 & 6,3 & 7,0 & 10,3 & - & 11,2 & 11,2 & 12,0 & 15,8 \\
\hline 10 & 3,9 & 6,8 & - & - & 11,6 & 16,2 & 16,5 & 16,1 \\
\hline 20 & 1,0 & 3,3 & 4,4 & - & 4,8 & 5,8 & 6,3 & 7,7 \\
\hline 30 & 0,3 & 0,7 & 1,2 & - & 0,9 & 3,7 & 1,1 & 1,7 \\
\hline
\end{tabular}

Die größte Zuwachsleistung oberirdischer Teile liegt im Langtag von 19 und 24 Std. bei $10 \%$ KM und 15500 Lux bzw. 5600 Lux (24 Std.-Tag) vor (Tab. 1). Im Grundsatz gilt bei allen KM-Konzentrationen, daß der Zuwachs oberirdischer Teile mit steigender Tageslänge und höherer Lichtintensität ansteigt.

Die Erhöhung der Festigkeit aller Triebe mit Erhöhung der Lichtintensität ergibt sich aus den mittleren Wassergehalten der oberirdischen Teile (Tab. 2), die bei allen Tageslängen jeweils bei der höheren Lichtintensität und mit steigender Tageslänge abnehmen. Der höchste Anteil der Trockensubstanz und damit die größte Festigkeit der Triebe und die höchste Zuwachsleistung oberirdischer Teile wird somit im Langtag erreicht. 


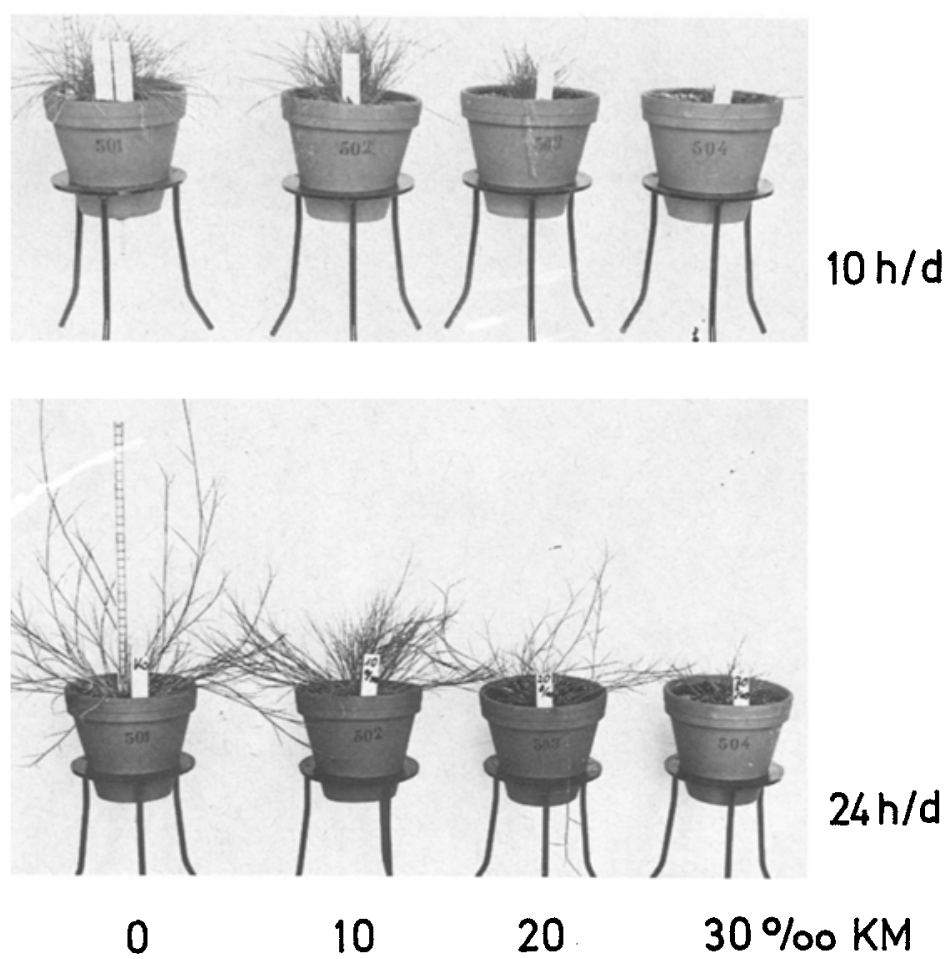

Abb. 2: Entwicklung von Puccinellia maritima (Sort.-Nr. 57) in Abhängigkeit von der Tageslichtdauer und der Meersalzkonzentration der Gießlösung nach 56 Tagen Kultur in einer Klimakammer $\left(20^{\circ} \mathrm{C}, 70 \%\right.$ rel. Luftfeuchte, 15500 Lux $)$

Tabelle 2

Mittlere Wassergehalte (\%) von Puccinellia maritima (Sort.-Nr. 57) in Abhängigkeit von der Tageslänge, der Lichtintensität und der Meersalzkonzentration der Gießlösung nach 56tägiger Kultur bei $20^{\circ} \mathrm{C}$ Lufttemperatur und $70 \%$ relativer Luftfeuchte in einer Klimakammer

\begin{tabular}{|lccccccccc|}
\hline $\begin{array}{l}\text { Belichtung } \\
\text { Std/Tag }\end{array}$ & \multicolumn{2}{c}{10} & \multicolumn{2}{c}{16} & \multicolumn{2}{c}{19} & \multicolumn{2}{c}{24} \\
Lux & 5600 & 15500 & 5600 & 15500 & 5600 & 15500 & 5600 & 15500 \\
\hline$\%$ KM & & & & & & & & \\
0 & 84,4 & 77,0 & 73,7 & - & 76,3 & 63,7 & 63,6 & 55,6 \\
10 & 82,6 & 79,3 & - & - & 79,0 & 65,9 & 67,5 & 55,4 \\
20 & 77,6 & 76,9 & 80,2 & - & 79,4 & 71,3 & 70,0 & 58,4 \\
30 & 70,0 & 75,0 & 75,8 & - & 78,1 & 71,9 & 62,6 & 58,2 \\
\hline
\end{tabular}

Darüber hinaus hat sich ergeben, daß die Entwicklung von Lang- und Kurztrieben abhängig von der Tageslänge ist (Abb. 3). Im Dauerlicht wurden ausschließlich Langtriebe der Typen 2a und $2 \mathrm{~b}$ entwickelt, im Kurztag von $10 \mathrm{Std}$. ausschließlich Kurztriebe des Typs 1a. Bei den Tageslängen von 16 bis 19 Std. traten ebenfalls Langtriebe der Typen 2a und $2 \mathrm{~b}$ aber mit reduzierter Zuwachsleistung auf. 


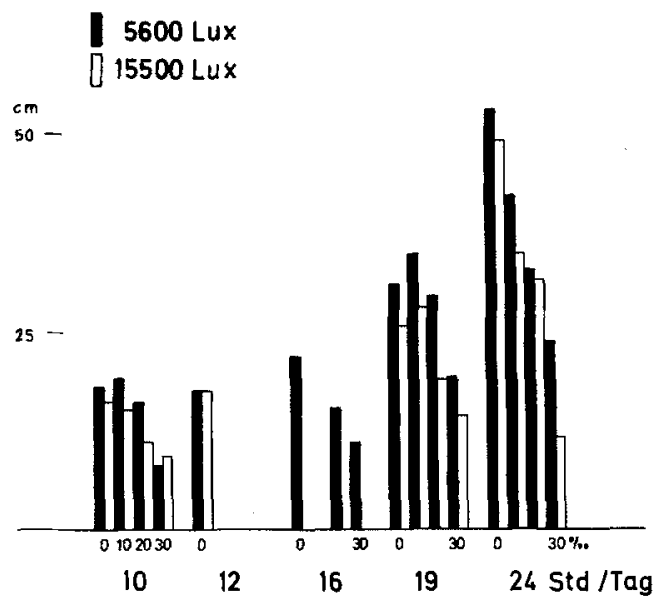

Abb. 3: Trieblängen von Puccinellia maritima (Sort.-Nr. 57) in Abhängigkeit von der Tageslichtdauer und der Meersalzkonzentration der Gießlösung nach 56 Tagen Kultur in einer Klimakammer $\left(20^{\circ} \mathrm{C}\right.$, $70 \%$ rel. Luftfeuchte)

Hiernach ist die Langtriebentwicklung an Tageslängen von 16 und mehr Stunden gebunden und eine Folge des Langtages, während die Kurztriebentwicklung eine Folge des Kurztages ist. Die Bestockungstriebe folgen offenbar den gleichen Gesetzmäßigkeiten, wonach die Länge der jeweiligen Langtriebe quantitativ den herrschenden Tageslängen folgt. Hiernach erscheint die Triebentwicklung mit ihrer Typisierung als quantitativer Tageslängeneffekt.

\section{Überflutung}

Die Versuche zur Feststellung der Uberflutungsverträglichkeit von Puccinellia maritima (Sort.-Nr. 57) wurden in einer Flutanlage mit Seeschlick und mit Quarzkies-Torf im Prinzip in der früher beschriebenen Weise (von Weihe \& Dreyling, 1970) durchgeführt. Die ebendort berichteten Teilergebnisse beziehen sich auf die Zuwachsleistungen einer Quarzkies-Torf-Kultur.

Die Entwicklung der Pflanzen nach 96tägiger Kultur ließ bei der Hochwasserhöhe von $1 \mathrm{~cm}$ über Bodenoberfläche das Optimum der Zuwachsleistung erkennen (Abb. 4). Hier wurde sowohl auf Quarzkies-Torf als auch auf Seeschlick die größte Zuwachsleistung der oberirdischen Pflanzenteile, die höchste Anzahl der Langtriebe und die größte Trieblänge pro Kulturgefäß erreicht (Tab. 3). Der hierbei vorliegende Hochwasserstand entspricht somit der MThw.

Die Differenzierung der Zuwachsleistung zwischen den unterschiedlichen Kultursubstraten betrifft in erster Linie die Frischgewichte und die Anzahl der entwickelten Triebe; hiernach ist das Optimum der Zuwachsleistungen auf Quarzkies-Torf-Substraten breiter als das auf Seeschlick-Substrat ausgeprägt.

Die in Tabelle 3 wiedergegebenen Bodenwassergehalte wurden unmittelbar vor Einsetzen der Flut, d. h. ca. 10-11 Std. nach Ablaufen des Wassers der davor liegenden 

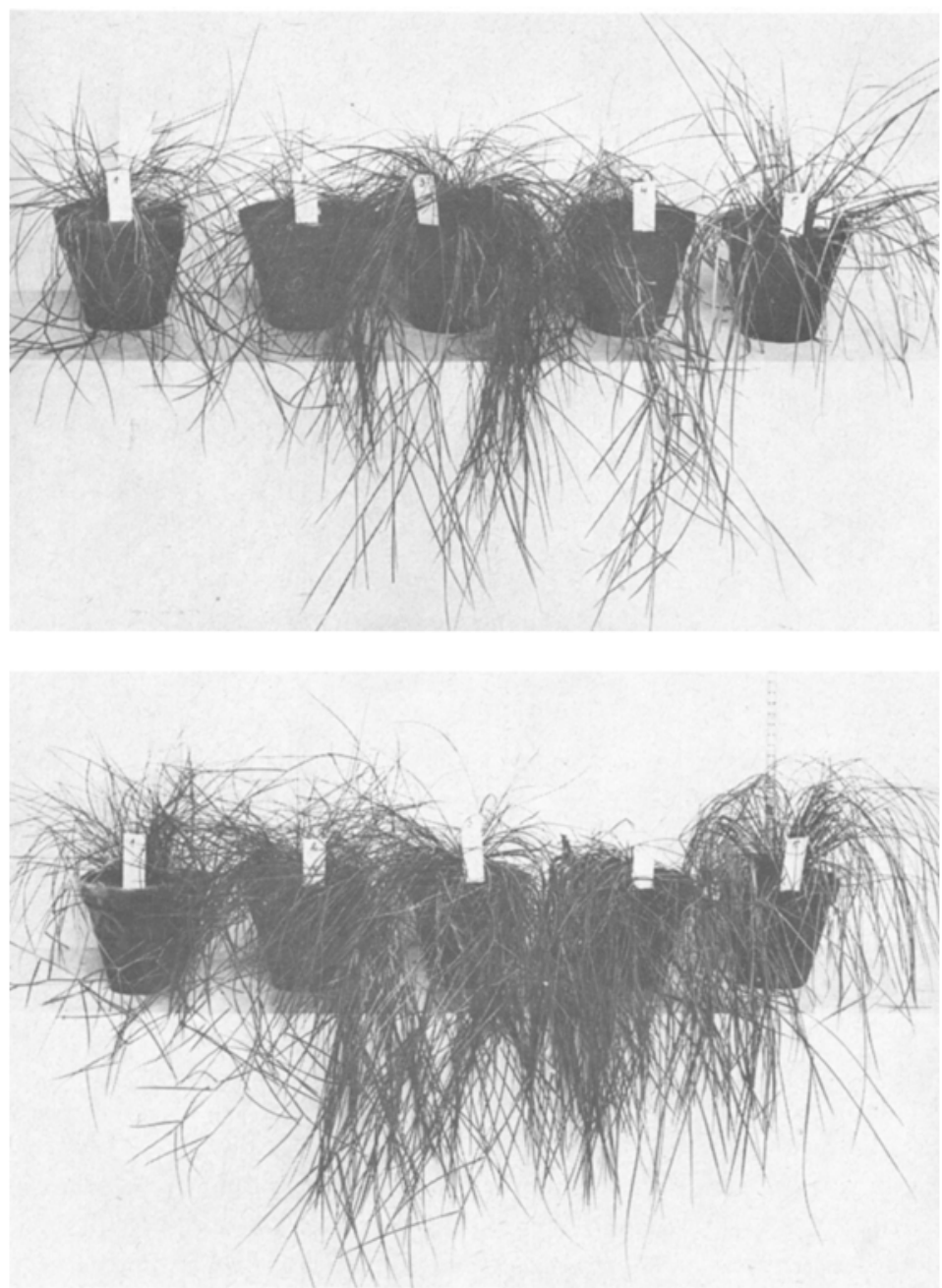

Abb. 4: Entwicklung von Puccinellia maritima (Sort.-Nr. 57) in Abhängigkeit von der Dauer der Überflutung . 12-Std.-Rhythmus) mit $25 \%$ KM nach 96tägiger Kultur auf Seeschlick- (obere Reihe) und Quázzkies-Torf-Substraten (untere Reihe) in einer Flutanlage im Gewächshaus (von links nach rechts Zunahme der Fluthöhen: $-7,-3,+1,+9,+18 \mathrm{~cm}$ unter/über Topfrand)

Flut gemessen. Hier ergibt sich - wie zu erwarten - daß der Bodenwassergehalt in Seeschlick höher als in Quarzkies-Torf ist und hier in den untersten Stufen der Flutanlage mit über $40 \%$ vorliegt und in den höchsten Stufen, in denen nur ein kapillarer Aufstieg des Meerwassers im Boden erfolgt, bis auf $29 \%$ absinkt. Im Gegensatz hierzu liegen im Quarzkies-Torf offenbar bessere Bodenbelüftungen in allen Stufen durch eine rel. geringe Bodenwassergehaltsdifferenz zwischen 27 und $22 \%$ vor. Die Feldkapazität wurde somit nur um $\sim 2-7 \%$ unterschritten. Diese letzte Tatsache ist vermutlich die Ursache für die relative breite Ausprägung des Optimums bei der Quarzkies-Torf-Kultur. 
Infolge des gewählten Kulturzeitraumes während der Vegetationsperiode lagen in allen Höhenstufen Bestockungen mit der Entwicklung von Langtrieben vor.

Zusammenfassend ergibt sich, daß hiernach die Höhe und die damit verbundene Dauer der Überflutung neben dem Frischgewichtszuwachs die Anzahl und damit die Entwicklung von Bestockungstrieben mitbeeinflußt. Langdauernde und fehlende Überflutungen setzen die Anzahl der Bestockungstriebe herab.

\section{Tabelle 3}

Mittlere Frischgewichte, Wassergehalte, Anzahl Triebe und Trieblängen von Puccinellia maritima (Sort.-Nr. 57) in Abhängigkeit von der Uberflutungsdauer und -höhe je Tide nach 96 Tagen Kultur (= 192 Uberflutungen) auf Quarzkies-Torf und Seeschlick im Gewächshaus bei $25 \% \mathrm{KM}$ des Flutwassers (Zahlenangaben z. T. nach von Weihe \& Dreyling, 1970)

\begin{tabular}{|c|c|c|c|c|c|}
\hline $\begin{array}{l}\text { Hochwasserstände } \\
\text { über/unter }(\mathrm{cm}) \\
\text { Bodenoberfläche }\end{array}$ & +18 & +9 & +1 & -3 & -7 \\
\hline $\begin{array}{l}\text { Uberflutungsdauer } \\
\text { pro Tide (min) }\end{array}$ & 204 & 141 & 72 & 0 & 0 \\
\hline $\begin{array}{l}\text { Seeschlick: } \\
\text { Bodenwassergehalt }(\%) \\
\text { Frischgewicht }(\mathrm{g}) \\
\text { Wassergehalt }(\%) \\
\text { Triebe (Anzahl) } \\
\text { Trieblänge }(\mathrm{cm})\end{array}$ & $\begin{array}{l}43,5 \\
15,4 \\
82,2 \\
51 \\
39,7\end{array}$ & $\begin{array}{l}46,2 \\
13,8 \\
79,3 \\
47 \\
46,5\end{array}$ & $\begin{array}{l}40,0 \\
25,6 \\
72,8 \\
90 \\
47,0\end{array}$ & $\begin{array}{r}35,3 \\
8,6 \\
66,6 \\
42 \\
33,5\end{array}$ & $\begin{array}{c}29,3 \\
7,6 \\
67,6 \\
27 \\
32,2\end{array}$ \\
\hline $\begin{array}{l}\text { Quarzkies-Torf: } \\
\text { Bodenwassergehalt }(\%) \\
\text { Frischgewicht }(\mathrm{g}) \\
\text { Wassergehalt }(\%) \\
\text { Triebe (Anzahl) } \\
\text { Trieblänge }(\mathrm{cm})\end{array}$ & $\begin{array}{l}26,0 \\
17,2 \\
82,2 \\
57 \\
40,4\end{array}$ & $\begin{array}{l}23,5 \\
25,9 \\
81,7 \\
85 \\
46,8\end{array}$ & $\begin{array}{c}27,8 \\
36,0 \\
76,3 \\
106 \\
53,2\end{array}$ & $\begin{array}{l}24,5 \\
21,3 \\
73,5 \\
85 \\
47,6\end{array}$ & $\begin{array}{r}22,0 \\
4,7 \\
70,6 \\
32 \\
26,0\end{array}$ \\
\hline
\end{tabular}

\section{Übersandung}

Die Gesamtentwicklung von Puccinellia maritima am natürlichen Standort steht neben der Morphologie, der Salz- und Überflutungsverträglichkeit der Art in engem Zusammenhang mit der Úberschlickung oder Übersandung. Nach Jakobsen et al. (1956) werden von $P$. maritima Uberschlickungen mit sandigem Ton bis zu $10 \mathrm{~cm}$ Höhe pro Jahr erțragen.

Zur Klärung der Ubersandungsverträglichkeit wurde bei den Versuchen mit $P$. maritima ebenso wie bei den Untersuchungen zur Ubersandungsverträglichkeit von Deschampsia wibeliana (von Weihe $\&$ Reese, 1968) grundsätzlich davon ausgegangen, daß nach einer Ubersandung Teile der Photosyntheseflächen der Testpflanze über die erhöhte Bodenoberfläche reichen müssen.

Aus diesen Gründen wurden alle Testpflanzen von P. maritima 94-97 Tage vor der Ubersandung von März bis Juni, bis zur größten Tageslänge vorkultiviert und die in dieser Zeit entwickelten Ausläufer bei der Übersandung über. die neue Bodenoberfläche geführt 
und hiernach 95 Tage weiterkultiviert. Die Entwicklung und der Verlauf der Triebentwicklungen der hier verwendeten Testpflanzen ist von Petermann (1970) eingehend erfaßt worden; hiernach lag am 94.-97. Tag der Vorkultur eine mittlere Langtrieblänge von $62 \mathrm{~cm}$ vor, so daß zum Zeitpunkt der Übersandung die folgenden mittleren Langtrieblängen oberhalb der neuen Bodenoberfläche vorlagen:

$10 \mathrm{~cm}$ Ubersandung: $52 \mathrm{~cm}$ nicht übersandete Langtriebe

$20 \mathrm{~cm}$ Ubersandung: $42 \mathrm{~cm}$ nicht übersandete Langtriebe

$30 \mathrm{~cm}$ Ubersandung: $32 \mathrm{~cm}$ nicht übersandete Langtriebe.

Tabelle 4

Mittlere Frischgewichte/Kulturgefäß, Wassergehalt und Länge der Ausläufer von Puccinellia maritima (Sort.-Nr. 57) nach 95 Tagen Ubersandungskultur im Gewächshaus (Flutverfahren mit $5 \% \mathrm{KM}$ )

\begin{tabular}{|lrrrr|}
\hline Ubersandungshöhe $(\mathrm{cm})$ & 0 & 10 & 20 & 30 \\
\hline Mittleres Frischgewicht (g) & 369,7 & 303,1 & 145,4 & 214,8 \\
Mirtlere Länge Ausläufer (cm): & & & & \\
Gesamtläge & 154,9 & 121,1 & 119,5 & 141,5 \\
Länge nach Ubersandung & 154,9 & 131,1 & 139,5 & 171,5 \\
Wassergehalt (\%) & 71,4 & 70,6 & 62,5 & 75,0 \\
\hline
\end{tabular}
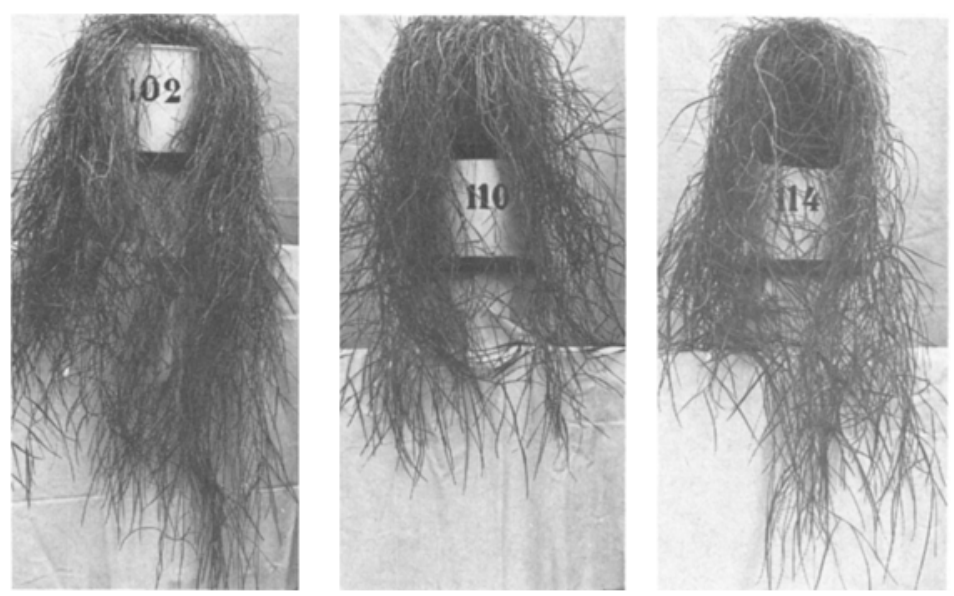

Abb. 5: Entwicklung von Puccinellia maritima (Sort.-Nr. 57) nach Úbersandung mit Quarzkies-Torf in Höhe von 10 (links), 20 (Mitte) und $30 \mathrm{~cm}$ (rechts) nach 192 Tagen Kultur im Flutverfahren mit $5 \% \mathrm{KM}$ im Gewächshaus

Nach Ubersandung verlief die Langtriebentwicklung weiterhin ähnlich wie bei den nicht übersandeten Testpflanzen (Abb. 5). Der Frischgewichtszuwachs verminderte sich nach der Ubersandung, die Gesamtlänge der Langtriebe überstieg bei $30 \mathrm{~cm}$ Übersandung die Gesamtlänge der Ausläufer der nicht übersandeten Pflanzen (Tab. 4).

Die Morphologie der übersandeten Abschnitte der Langtriebe wies z. T. Achsenstrekkungsabschnitte an Bestockungstrieben auf, die zur Hebung der Vegetationskegel bis in die Nähe der neuen Bodenoberfläche führte. Im übrigen erfolgte eine umfangreiche Ausbildung von Adventivwurzeln an den übersandeten Knoten der Langtriebe (Abb. 6). Die 

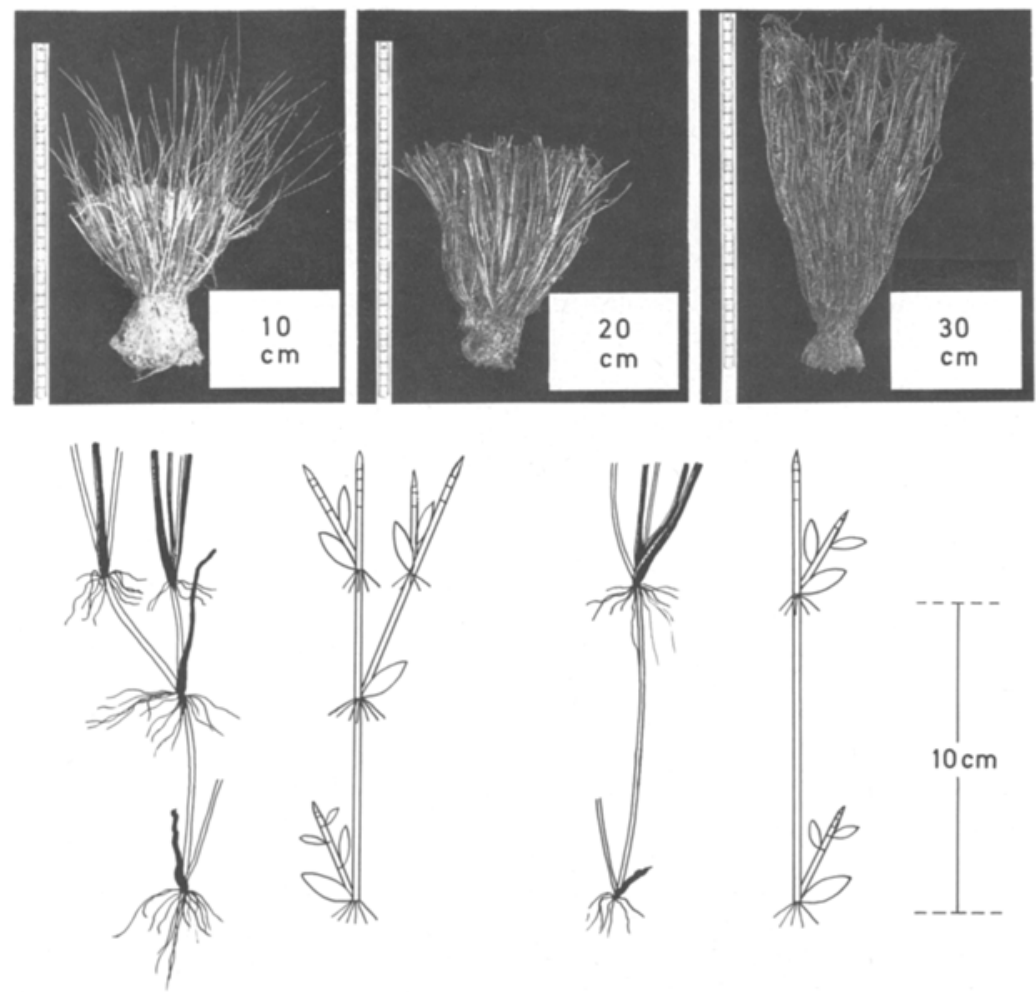

Abb. 6: 10, 20 und $30 \mathrm{~cm}$ hoch übersandete Triebe von Puccinellia maritima (Sort.-Nr. 57) und morphologische Analyse nach 95 Tagen Ubersandungskultur im Flutverfahren mit $5 \%$ KM im Gewächshaus

Bestockungstriebe mit Achsenstreckung entsprechen dem Übersandungstyp, der auch mit Deschampsia wibeliana vorliegt.

Bei der Ernte wurde bei allen Ubersandungsknoten festgestellt, daß nur ein Teil der übersandeten Triebe und Wurzeln abgestorben waren; an der Wasser- und Nährstoffleitung dürften somit bei diesem gut durchlüfteten Übersandungssubstrat auch die älteren übersandeten Ausläuferteile mitbeteiligt sein.

Die Adventivwurzelbildung an den Knoten der Langtriebe trat im übersandeten Sproßteil oder kurz oberhalb der Bodenoberfläche auf; Langtriebe, die sich oberhalb des Bodens entwickelten und dem Boden nicht auflagen, bildeten entweder keine Adventivwurzeln aus oder die in Bodennähe im Luftraum an den Langtrieben ohne Bodenberührung entwickelten Adventivwurzeln vertrockneten zum größten Teil.

\section{GESAMTAUSWERTUNG UND DISKUSSION}

Die Ergebnisse der berichteten experimentellen Untersuchungen haben u. a. zu den folgenden Merkmalen von Puccinellia maritima geführt: 
(1) Die Längenentwicklung der Bestockungstriebe ist $z$. T. eine quantitative Reaktion auf die vorliegende Tageslichtdauer, nach der im Prinzip im Langtag ortho- oder plagiotrope Langtriebe und im Kurztag unterhalb einer kritischen Tageslichtdauer (vermutlich 12 Std.) Kurztriebe entwickelt werden, die die Ursache für den Saisondimorphismus (Ausläufergras - Horstgras) darstellen.

(2) Die Dauer und die Höhe der Uberflutung beeinflussen den Zuwachs der oberirdischen Pflanzenteile. Die größte Zuwachsleistung wird bei einer Úberflutungshöhe erreicht, die den Höhen der MThw des natürlichen Standortes vergleichbar ist.

(3) Eine Ubersandung der Langtriebe wird unter der Voraussetzung, daß nach der Übersandung distale grüne Pflanzenteile über die Bodenoberfläche herausragen, mindestens bis zu einer Höhe von $30 \mathrm{~cm}$ ertragen. Eine Utbersandung führt in der Regel dazu, daß sich an den von der Übersandung betroffenen Knoten der Langtriebe Adventivwurzeln ausbilden.

Diese Merkmale sind im Zusammenhang u. a. mit den Ergebnissen der Untersuchungen über die Meersalzwirkungen (von Weihe \& Dreyling, 1970; von Weihe, 1978) zu diskutieren.

Die Ausbildung plagiotroper und orthotroper Langtriebe ist z. T. topographisch bedingt; die plagiotropen Langtriebe stellen "Kriechtriebe" im Sinne von Mühlberg (1967) dar. Die Entwicklung dieser Triebe, ihre Bestockung und Adventivwurzelbildung ist eingehend von Petermann (1970) untersucht worden. Hiernach erfolgte in ihrem Untersuchungszeitraum vom März bis Oktober (Tageslichtdauer: 12,8-18,5-12,2 Std.) eine Steigerung des Längenzuwachses der Ausläufer durch hohe Temperaturen. Die größte tägliche Zuwachsrate wurde bei ihren Testpflanzen zwischen dem 13.6. und dem 21. 7. beobachtet, die niedrigsten täglichen Zuwachslängen lagen mit $\leqslant 0,1 \mathrm{~mm}$ ab 25. 8. vor. Die Kurztriebentwicklung setzte verstärkt im August ein, so daß bis zu $71 \%$ der neu hinzugekommenen Triebe als Kurztriebe ausgebildet waren. Bei den dort horizontal auf Quarzkies-Torf kultivierten plagiotropen Ausläufern traten zu Versuchsende (bis 9. Oktober) typische orthotrop wachsende Kurztriebe in den Achseln der Blätter der Langtriebe auf, und die Spitzen einiger plagiotroper Langtriebe richteten sich auf und entwickelten an der Spitze einen orthotropen "Kurztrieb".

Diese Ergebnisse decken sich weitgehend mit den hier vorgelegten Versuchsergebnissen zur Untersuchung der Wirkung der Tageslichtdauer und weisen aus, daß eine Internodienstreckung am gleichen Trieb von der Tageslänge und der Temperatur beeinflußt wird, daß also der gleiche Trieb periodisch als Lang- oder Kurztrieb wachsen kann. Die quantitative Wirkung der Tageslichtlänge ist dagegen offenbar infolge der Temperaturwirkung nur im ausgeprägtesten Langtag und im Kurztag zum Ende der Versuche deutlich, zeichnet sich aber im sigmoiden Verlauf der Längenentwicklungskurve der Langtriebe bei Petermann (1970) mit individuellen Unterschieden ab. Jene Versuche wurden mit $5 \%$ KM durchgeführt.

Die eigenen Versuche weisen aus, daß der Längenzuwachs neben der Temperatur (von Weihe, 1978) von dem Meersalzgehalt des Flutwassers mit beeinflußt wird. Steigende KMKonzentration - je nach vorliegendem Salinitätsoptimumtyp $->0$ bzw. $>5-10 \%$ reduzieren den Zuwachs.

Diese Langtagwirkung auf die Triebentwicklung ist vermutlich auch bei anderen 
Gräsern mit oberirdischen Ausläufern ausgeprägt und in gewissem Umfange vergleichbar mit der Ausbildung der Ausläufer bei Fragaria $X$ ananassa (Darrow, 1937).

Dauer und Höhe der Uberflutung mit Meerwasser wirken sich nach den hier vorgelegten Versuchsergebnissen ebenfalls auf die Zuwachsleistung von $P$. maritima entscheidend aus. Das hier ermittelte Optimum der Zuwachsleistung liegt praktisch etwa in Höhe der MThw vor. Dieses Optimum liegt an der oberen Grenze des von Neugebohrn (1974) ermittelten Optimums der Karyopsen-Keimung von P. maritima in Abhängigkeit von Fluthöhe und -dauer. Nach den Analysen der natürlichen Standorte der Art im Puccinellietum maritimae nach von Weihe \& Dreyling (1970) steht die Verbreitung vermutlich zusätzlich im Zusammenhang mit der Uberflutungshäufigkeit.

Außerhalb des Optimums ist die Zuwachsleistung der oberirdischen Pflanzenteile herabgesetzt bzw. die Anzahl der Triebe vermindert. Dieses Optimum ist bei einer Kultur auf Seeschlick schmaler als bei einer Kultur auf Quarzkies-Torf ausgeprägt. Nach Gillner (1952, 1960) und von Weihe (1978) ist die Deckung von P. maritima am natürlichen Standort eindeutig mit der Pegelhöhe und der Anzahl der Úberflutungen und innerhalb des Bereiches des Puccinellion und Armerion nicht mit dem Meersalzgehalt der Bodenlösun-

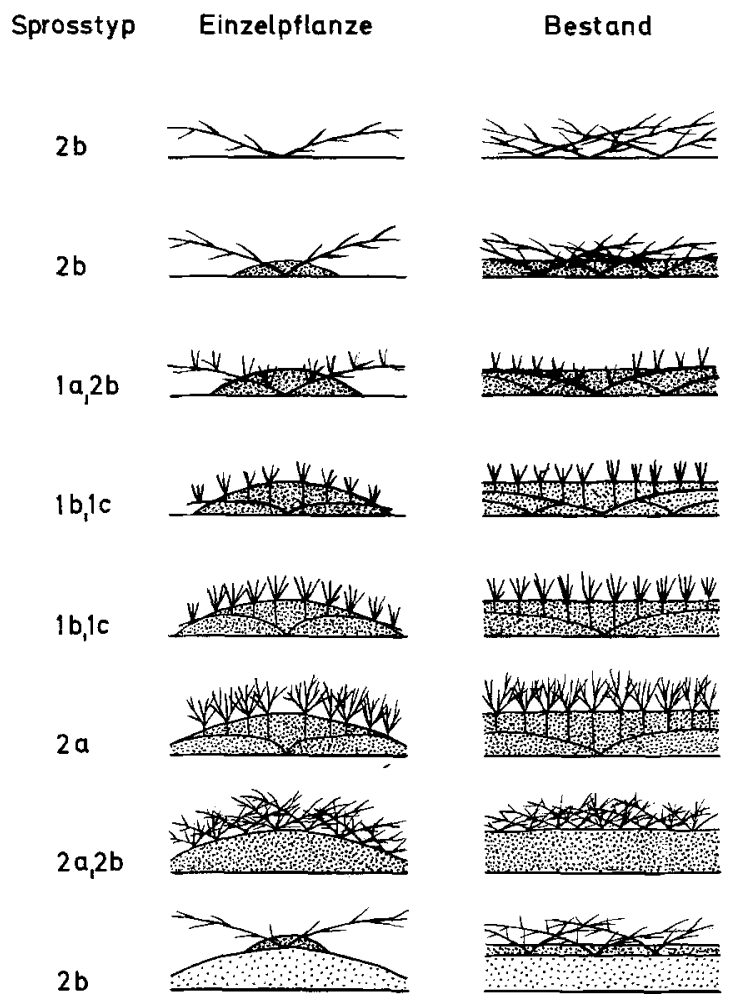

Abb. 7: Schematische Entwicklung von Puccinellia maritima mit und ohne Uberschlickung oder Ubersandung unter Berücksichtigung des Saisondimorphismus der Art (1a: Kurztrieb ohne Bestokkung, 1b: Kurztrieb mit Bestockung, 1c: Kurztrieb mit Achsenstreckung, 2a: orthotroper Langtrieb, 2b: plagiotroper Langtrieb) 
gen korreliert. Dies läßt nach den experimentellen Befunden auf einen kausalen Zusammenhang mit der Uberflutung schließen.

Der Umfang der Zuwachsleistung der Lang- und Kurztriebe einschließlich der damit verbundenen Blätterausbildung bestimmen - neben der Dichte der P. maritima-Bestände am natürlichen Standort durch die Schaffung von Stillwasserräumen den Umfang und die Korngröße der Sedimentation bei einer Uberflutung entsprechend dem KorngrößenFallzeiten-Modell nach Köhn (1928). Somit bestimmen diese Entwicklungen von $P$. maritima die entscheidenden Leistungen der Art und des Puccinellietum maritimae für den Sedimentfang. Für die allgemeinen Vorgänge bei der Sedimentation in den Salzwiesen ist auf die Angaben bei Ranwell (1975) hinzuweisen.

Diese Sedimentation führt zu einer partiellen Einbettung der Triebe in das Sediment. Nach Jakobsen et al. (1956) tritt bei $P$. maritima eine Uberschlickung bis zu $10 \mathrm{~cm}$ pro Jahr auf. Nach den hier vorgelegten Versuchsergebnissen wird $z$. T. mit erhöhter nachträglicher Zuwachsleistung eine Übersandung mit gut durchlüftetem Quarzkies-Torf bis zu $30 \mathrm{~cm}$ bei einer Kultur in $5 \% \mathrm{KM}$ ertragen.

Diese Übersandung führt einerseits zu einer verstärkten Adventivwurzelbildung im Bereich der übersandeten Langtriebe, andererseits zu einer Achsenstreckung von Kurztrieben, die zu einer Hebung der Triebspitze u. a. um $10 \mathrm{~cm}$ bis zur Höhe der Bodenoberfläche führen und im Prinzip ein orthotropes Wachstum voraussetzen. Die Umkehr der Wachstumsrichtung der Langtriebe in den Herbstmonaten von plagiotropem zu orthotropem Wuchs liefert eine Voraussetzung für eine Achsenstreckung nach winterlicher Übersandung oder Überschlickung.

Das Ubersandungsverhalten der Kurztriebe von $P$. maritima entspricht dem Vorgang der Achsenstreckung wie er nach Ubersandung z. B. bei Deschampsia wibeliana (von Weihe \& Reese, 1968), Puccinellia capillaris (von Weihe \& Dreyling, 1978) nachgewiesen oder auch von Ammophila arenaria, Corynephorus canescens etc. bekannt ist.

Unter Berücksichtigung der Ergebnisse der Konkurrenzanalysen bei der Bodenaussüßung der P. maritima-Soden (von Weihe, 1969) und der Untersuchungen zur Meersalzverträglichkeit der Art ergibt sich derzeit folgendes Bild des Zusammenwirkens der Standortfaktoren:

P. maritima hat als fakultativer Halophyt ihr physiologisches Optimum (im Sinne von Ellenberg, 1952) bei 0 oder 5-10\% Meersalzkonzentration der Bodenlösung. An diesen Standorten liegen gleichzeitig auch die Schwerpunkte der Konkurrenz durch andere Arten u. a. durch Uberwachsungs- und Unterwachsungstypen (im Sinne von Knapp \& Knapp, 1955) wie Lolium perenne, Poa pratensis und Festuca rubra dadurch vor, daß infolge des Saisondimorphismus von $P$. maritima orthotrope und plagiotrope Ausläufer bei einem dichten Bestand konkurrierender Arten nicht mehr der Bodenoberfläche aufliegen; diese Langtriebe und die später vorliegenden Kurztriebe können sich nicht bewurzeln; die Triebe wintern aus.

P. maritima besitzt eine breitere Salzverträglichkeitsamplitude und eine größere Uberflutungsverträglichkeit als die genannten Arten, insbesondere als Festuca rubra ssp. litoralis. Hierdurch besiedelt $P$. maritima in ihrem ökologischen Optimum die relativ konkurrenzarme Zone in Höhe und wenige Dezimeter oberhalb der MThw.

An diesem Standort besteht für P. maritima selbst als Überdauerungstyp und $z$. T. auch als Überwachsungstyp (im Sinne von Knapp \& Knapp, 1955) erhöhte Konkurrenz- 
kraft der Art gegenüber der einjährigen und ausgeprägten Sonnenpflanze Salicornia stricta, deren physiologische Amplitude zwar bis ins Puccinellietum reicht, deren ökologisches Optimum aber unterhalb der MThw liegt. In dieser Zone kurz oberhalb der MThw entwickelt sich ein Konkurrenzgleichgewicht von $P$. maritima mit weiteren Arten, eben die Ausbildung des Puccinellietum maritimae.

Die oben genannte, morphologisch bedingte Konkurrenzschwäche von $P$. maritima gegenüber den höher in der Salzwiese oder in der Fettwiese siedelnden Arten wird in der Höhe der MThw durch die Trieb- und Blattproduktion von P. maritima selbst mittels Sedimentfang, durch die Sedimentation von Bodenpartikeln in Stillwasserzonen des Überflutungswassers kompensiert.

Ubersandung und Uberschlickung, die Erhöhung der Bodenoberfläche wird durch den Saisondimorphismus und die Achsenstreckung ausgeglichen; sie führt zur Wurzelbildung an den Langtrieben und zur Achsenstreckung und zur Bewurzelung orthotroper Kurztriebe; beides zieht eine verstärkte Sedimentbindung, die entscheidende Voraussetzung zur Bodensicherung in der unteren Salzwiese, nach sich.

Hiermit leitet die Art die allogene Sukzession ein. P. maritima stellt quasi selbst das abbauende Element des Puccinellietum maritimae durch die Steigerung der Sedimentationsrate dar, die gleichzeitig durch die Bodenerhöhung zu einer Vitalitätsminderung von $P$. maritima gegenüber den Arten des Juncetum gerardii führt. Die Triebtypenfolge von $P$. maritima im Laufe eines Jahres in der Zone des ökologischen Optimums (Abb. 7) ist somit zur Differenzierung wie folgt zu ergänzen:

Triebtypen: (1) vernale und autumnale Kurztagtypen

(1a) orthotroper, nicht bestockter Kurztrieb

(1b) orthotroper, bestockter Kurztrieb

(1c) orthotroper, Kurztrieb mit Achsenstreckung

(2) aestivale Langtagtypen

(2a) orthotroper Langtrieb

(2b) plagiotroper Langtrieb.

Abbildung 7 gibt die Zusammenhänge zwischen den Triebtypen und der Bodenaufhöhung wieder. Hiernach tritt in geschlossenem Bestand von $P$. maritima eine gleichmäßige Bodenerhöhung durch die ineinandergreifenden Langtriebe des Typs $2 b$ und eine VergröBerung des Stillwasserraumes bei Uberflutung durch die Typen 2a und $2 b$ auf; nach der Bodenerhöhung führt im Kurztag der Typ 1c zu einer Erhöhung des Bestockungsniveaus bis zur jeweils vorliegenden Bodenoberfläche unter der Schaffung von Stillwasserräumen. Der Saisondimorphismus von $P$. maritima ist somit die entscheidende Voraussetzung für den Sedimentfang, die Sedimentbindung und damit für die Bodenerhöhung und für Bodenund Vorlandsicherung in der unteren Salzwiese.

Danksagungen: Die langfristige Durchführung dieser Untersuchungen ist nur durch die Hilfe und den Einsatz vieler Mitarbeiter an den natürlichen Standorten, in den Gewächshäusern und Klimakammern und im Labor möglich gewesen. Den Damen Frau Dr. G. Dreyling, Frau B. Nossag geb. Petermann, Fräulein M. Hilbrig, Fräulein I. Oncken und den Herren Dr. L. Neugebohrn, H. Günther, G. Jonas, J. Schering und seinen Mitarbeitern auf dem Versuchsfeld des Instituts und B. Heitmann gilt u. a. besonderer Dank. 


\section{ZITIERTE LITERATUR}

Darrow, G. M., 1937. Interrelation of temperature and photoperiodism in the production of fruitbuds and runners in the strawberry. Proc. Am. Soc. hort. Sci. 34, 360-363.

Dreyling, G., 1973. Spezifische und infraspezifische Mannigfaltigkeit der Gattung Puccinellia Parlatore (Poaceae) von der deutschen Nordseeküste. Diss. Hamburg, $150 \mathrm{pp}$.

Ellenberg, H., 1952. Physiologisches und ökologisches Verhalten derselben Pflanzenarten. Ber. dt. bot. Ges. 65, 350-361.

Gillner, V., 1952. Die Gürtelung der Strandwiesen und der Wasserstandswechsel an der Westküste Schwedens. Svensk. bot. Tidskr. 46, 393-428.

- 1960. Vegetations- und Standortuntersuchungen in den Strandwiesen der schwedischen Westküste. Acta phytogeogr. suec. 43, 1-198.

Gray, A. J. \& Scott, R., 1975. In: A. Rep. Institute of Terrestrial Ecology Norwich 1973-1974, 36.

Hudson, W., 1762. Flora Anglica. London.

Jacobson, L., 1951. Maintenance of iron supply in nutrient solutions by a single addition of ferric potassium ethylenediamine tetra-acetate. Pl. Physiol., Lancaster 26, 411-413.

Jakobsen, B., Jensen, K. M. \& Nielsen, N., 1956. Forslag til landvindingsarbejder langs den sømderjyske Vadehavskyst. Geogr. Tidsskr. 55, 62-87.

Knapp, G. \& Knapp, R., 1955. Uber Möglichkeiten der Durchsetzung und Ausbreitung von Pflanzenindividuen auf Grund verschiedener Wuchsformen. Ber. dt. bot. Ges. 67, 410-419.

Köhn, M., 1928. Beiträge zur Theorie und Praxis der mechanischen Bodenanalyse. Landw. Jb. 67, $485-546$.

Levring, T., 1946. Some culture experiments with $U l v a$ and artificial sea water. K. fysiogr. Sällsk. Lund Förh. 16, (7) 45-56.

Mühlberg, H., 1967. Die Wuchstypen der mitteldeutschen Poaceen. Hercynia (Lpz.) N. F. 4, 11-50.

Neugebohrn, L., 1974. Beiträge zur Okologie der Karyopsen-Keimung von Puccinellia maritima (Huds.) Parl. (Grundlagen zur Frage der Anwendung der Art bei planmäßigen Aussaaten im Küstenschutz). Diss. Hamburg, 98 pp.

Petermann, B., 1970. Anatomie und Morphologie der Ausläufer von Puccinellia maritima (Huds.) Parl. in Abhängigkeit vom Standort. Dipl.-Arb., Hamburg, 109 pp.

Ranwell D. S., 1975. Ecology of salt marshes and sand dunes. Chapman \& Hall, London, 258 pp.

Schratz, E. \& Beiler, A., 1937. Beobachtungen über die Salzverhältnisse und Halophytenvegetation im Adolf-Hitler-Koog. Ber. dt. bot. Ges. 55, 506-513.

Weihe, K. von, 1963. Untersuchungen zur Okologie und Morphologie von Festuca rubra L. ssp. rubra (Temperatur und Meersalzwirkung). Beitr. Biol. Pfl. 39, 239-262.

- 1969. Konkurrenzvorgänge bei der Aussüßung von Soden des Puccinellion maritimae. Die Küste (im Druck).

- 1978. Untersuchungen zur Ókologie von Puccinellia maritima (Huds.) Parl. (Temperatur und Meersalzwirkung). Beitr. Biol. Pfl. 54, 145-163.

— \& Dreyling, G., 1970. Kulturverfahren zur Bestimmung der Salz- und Überflutungsverträglichkeit von Puccinellia spp. (Gramineae). Helgoländer wiss. Meeresunters. 20, 157-171.

- \& Reese, G., 1968. Deschampsia wibeliana (Sonder) Parlatore. Beiträge zur Monographie einer Art des Tidegebietes. Bot. Jb. 88, 1-48.

Wohlenberg, E. 1931. Die Grüne Insel in der Eidermündung. Eine entwicklungs-physiologische Untersuchung. Aus Arch. dt. Seew. 50 (2), 1-34.

- 1933. Das Andelpolster und die Entstehung einer charakteristischen Abrasionsform im Wattenmeer. Wiss. Meeresunters (Helgoland) 19 (4), 1-10.

- 1969. Landgewinnung an der Küste durch biogene Verlandung. Handbuch für Landschaftspflege und Naturschutz. Hrsg. von K. Buchwald \& W. Engelhardt. Bd. 4: Planung und Ausführung. BLV-Verl.Ges., München, 196-204. 